\title{
Limit Laws for Terminal Nodes in Random Circuits with Restricted Fan-Out: A Family of Graphs Generalizing Binary Search Trees
}

\author{
Hosam Mahmoud ${ }^{1} \quad$ Tatsuie Tsukiji ${ }^{2}$
}

\begin{abstract}
We introduce a family of graphs $C(n, i, s, a)$ that generalizes the binary search tree. The graphs represent logic circuits with fan-in $i$, restricted fan-out $s$, and arising by $n$ progressive additions of random gates to a starting circuit of $a$ isolated nodes. We show via martingales that a suitably normalized version of the number of terminal nodes in binary circuits converges in distribution to a normal random variate.
\end{abstract}

Keywords: random graph, combinatorial probability.

\section{Introduction}

The well-known binary search tree has many applications as a data structure (see Mahmoud (1992)), as a model underlying searching and sorting applications (see Knuth 1998) and Mahmoud (2000)), and as a model for formal languages and computer algebra (see Kemp (1984)). We introduce a family of acyclic directed graphs $C(n, i, s, a)$ that generalizes the binary search tree.

The graphs represent logic circuits with indegree (fan-in) $i$, restricted outdegree (fan-out) $s$, arising by $n$ progressive additions of random gates to a starting circuit of $a$ isolated nodes. The initial $a$ nodes represent the initial input lines. The model has applications in neurosciences (see Valiant (1994)) and electrosciences (see Hutton, Rose, Grossman and Cornell (1998)).

The family of graphs $C(n, i, s, a)$ we introduce is a hierarchy of combinatorial structures that includes the binary search tree. We shall refer to a circuit with fan in $i$ and restricted fan-out $s$ as the $i$-ary circuit with fanout $s$. For specified parameters $i, s, a$, we refer to the structure simply as the circuit. Nodes with outdegree $s$ are considered saturated. At each stage, $i$ insertion positions are chosen from unsaturated nodes as the parents of a new child. The outdegree of each of these nodes increases by the number of insertion positions taken from it. In circuit interpretation, $i$ output lines of a previous stage are taken as input lines into a new $i$-ary gate, which can have up to $s$ output lines drawn from it. The consumed lines are no longer viable inputs at later stages.

Let $L_{n, i, s, a}$ denote the number of terminal nodes in the circuit having $a$ initial input nodes. The main result of this paper is to demonstrate the convergence in distribution of a suitably normed version of $L_{n, i, s, a}$ to a normal variate for binary circuits. The algebra gets quite unwieldy, if we keep all the parameters. For clarity of the exposition, we shall illustrate the result on the subfamily $C(n, 2,3,1)$ of binary fan-in, fan-out 3 , and growing out of a single node. Throughout all insertion stages, the underlying undirected graph remains one connected component; the fan-out is restricted to 3 . We shall return at the end to the general binary case and state the necessary adjustments in the proof to get a general result for the family $C(n, 2, s, a)$ and make some conjectures about what to expect in the class $C(n, i, s, a)$.

Throughout, we shall use the following notation. We shall denote the normally distributed random variate with mean 0 and variance $\sigma^{2}$ by $\mathcal{N}\left(0, \sigma^{2}\right)$. We shall use the symbols $\stackrel{\mathcal{D}}{\longrightarrow}$ and $\stackrel{P}{\longrightarrow}$ for convergence in distribution and in probability, respectively. The notation $O_{\mathcal{L}_{1}}(g(n))$ will stand for a random variable that is $O(g(n))$ in the $\mathcal{L}_{1}$ norm.

Let the notation $L_{n}$ be reserved for $L_{n, 2,3,1}$. One main result of this investigation is the central limit tendency:

$$
\frac{L_{n}-\frac{1}{7} n}{\sqrt{n}} \stackrel{\mathcal{D}}{\longrightarrow} \mathcal{N}\left(0, \frac{24}{637}\right),
$$

and its extension to a similar central limit theorem for $L_{n, 2, s, a}$.

Section 2 gives a precise definition for the circuits. In Section ?? we derive the exact first two moments for the number of terminal nodes for $C(n, 2,3,1)$. The central limit theorem for $C(n, 2,3,1)$ is derived in Section ?? via a martingale transform. The scope of the result and proof technique is extended in Section ?? to the binary circuits family $C(n, 2, s, a)$, where a conjecture for $C(n, i, s, a)$ is presented, too.

\footnotetext{
1 Department of Statistics, The George Washington University, Washington, D.C. 20052, U.S.A. E-mail address: hosam@gwu.edu

${ }^{2}$ Department of Information Sciences, Tokyo Denki University Hatoyama-cho, Saitama-ken 350-0394, Japan. E-mail address: tatsuie@ybb.ne.jp
} 


\section{The growth of a random circuit}

An $i$-ary circuit with fan-out $s$ is a directed graph that starts out with $a$ isolated nodes of indegree and outdegree 0 . Each node can have up to $s$ positional children (output lines in the language of circuits), with a distinguished left-to-right order. A childless node is a terminal node. A nonterminal node with $k$ children has $s-k$ positional insertion places, which are the positions not taken by its positional children in the left-to-right order. The circuit evolves in stages as follows. After $n-1$ stages, a circuit $C(n-1, i, s, a)$ has grown. At the $n$th stage, $i$ positional insertion places from unsaturated nodes are chosen from $C(n-1, i, s, a)$ as parents for a new node (or gate in the circuits). The new node is adjoined to the circuit with edges directed from the $i$ positional insertion places to it, and is given 0 outdegree.

With regard to the interpretation of $C(n, i, s, a)$ as a circuit, the number of terminal nodes is a parameter of interest. In boolean circuits they stand for how many "answers" can be derived from $a$ given inputs. The problem is trivial for $s=1$. The case $i=1$ is not very challenging. The least $i$ that makes the problem significant is 2 . The case $C(n, 2,2, a)$ is not too interesting, as the number of terminal nodes remains finite and at most $a$ throughout all the stages. The smallest significant member of the family $C(n, 2, s, a)$ for the study is $C(n, 2,3, a)$. For simplicity of the exposition we take $a=1$ to reduce the number of parameters. We shall focus on the binary circuit (fan-in $i=2$ ), with fan-out 3 , growing out of a single input. We develop results for $C(n, 2,3,1)$ in this section. In Section ?? we shall sketch the extension of these results to cover the entire $C(n, 2, s, a)$ family.

The graphs $C(n, i, s, a)$ generalize binary search trees (which are $C(n, 1,2,1)$ ). This runs in a parallel vein to the generalization of recursive trees into a hierarchy of random graphs called recursive circuits (Tsukiji and Mahmoud (2001)). For a broad review of definition and uses of recursive trees the reader is referred to the survey in Smythe and Mahmoud (1996).

For modeling and analysis purposes, the random graphs $C(n, i, s, a)$ are extended by supplying each node with a sufficient number of special nodes (called external nodes) to universally make the outdegree of all the circuit nodes (now viewed as internal) equal to $s$.

Many models of randomness can be imposed on $i$-ary circuits with fan-out $s$. A natural probability model is one in which all pairs of external nodes in $C(n-1, i, s, a)$ are equally likely candidate inputs for the $n$th entrant. Note that under this model, the various circuits of one size are not equally likely.

\section{References}

[1] Hall, P. and Heyde, C. (1980). Martingale Limit Theory and Its Applications. Academic Press, New York.

[2] Hutton, M., Rose, J., Grossman, J. and Cornell D. (1998). Characterization and Parameterized Generation of Synthetic Combinational Benchmark Circuits. IEEE Trans. on CAD, 17, 985-996.

[3] Kemp, R. (1984). Fundamentals of the Average Case Analysis of Particular Algorithms. WileyTeubner Series in Computer Science, Wiley, New York.

[4] Knuth, D. (1998). The Art of Computer Programming, Vol. 3: Sorting and Searching, 2nd ed. Addison-Wesley, Reading, Massachusetts.

[5] Mahmoud, H. (1992). Evolution of Random Search Trees. Wiley, New York.

[6] Mahmoud, H. (2000). Sorting: A distribution Theory. Wiley, New York.

[7] Smythe, R. and Mahmoud, H. (1996). A survey of recursive trees. Theory of Probability and Mathematical Statistics, 51, 1-29.

[8] Tsukiji, T. and Mahmoud, H. (2001). A limit law for outputs in random circuits. Algorithmica, 31, 403-412.

[9] Valiant, L. (1994). Circuits of the Mind. Oxford University Press, Oxford, UK. 